\title{
Descriptions of Reversed Yielding in Bending
}

\author{
D. W. A. Rees \\ School of Engineering and Design, \\ Brunel University, Uxbridge, \\ Middlesex UB8 3PH, UK.
}

\section{Summary}

Allowing for the Bauschinger effect, a second plastic penetration can occur with the release of the moment required for elastic-plastic bending of a beam. The theory is given for both linear and parabolic hardening material models. Within the hardening law the elastic and plastic strains are developed from the beam curvature of the unstressed neutral axis. Conditions are expressed in terms of a rectangular beam section and a normalised stressstrain response for which the release remains purely elastic and elastic-plastic. Under that latter the depth to which a second zone of plasticity penetrates is given. The two stress distributions: one for applying the moment and the other for its release, are sufficient to derive the residual stress. Residuals found for parabolic hardening are believed to be more realistic than those from simpler linear or perfectly plastic models particularly where a second penetration is evident.

\section{Introduction}

It is well known that the elastic-plastic bending of a beams results in a residual stress distribution when the moment is released. The idela elastic-perfectly plastic theory arrives at a solution to the residuals from assuming that plastic penetration into the depth occurs with no hardening as the moment is applied. Moreover, with its constant elastic range being twice the yield stress, the ideal material ensures that the moment release always remains elastic. However, in practice, plastic flow hardens real materials which, in reversed flow, reveal a reduced elastic range with an accompanying Bauschinger effect. The latter effect can reduce the elastic range to such an extent that a moment release can result in the penetration of a further zone of plasticity into the beam by reversed yielding. Though many studies have appeared on the subject of reversed yielding and the Bauschinger effect [1-4] this present application to beam bending has not been investigated before. This paper examines the 
reversed yielding phenomenon in the case of two further material models with linear and parabolic hardening. The accounts given show that the final residual stress distributions depend upon both forward and reversed flow curves and may attain maximum values up to $50 \%$ of the initial yield stress. It will be shown that the residuals under linear hardening with a second penetration do not differ appreciably from those of the ideal material. In contrast, the non-linear hardening model reflects more realistically the influence of Bauschinger effect upon the residual stresses.

\section{Stress and Strain Relationships}

Consider the three initially, elastically strained fibres $\mathrm{AB}, \mathrm{CD}$ and $\mathrm{EF}$ in a beam under bending (Fig. 1a). Let the moment be increased to the extent where the yield stress of the beam material $Y_{o}$ is reached in tension and compression at the outer fibre CD. The inner fibres $\mathrm{AB}$ and $\mathrm{EF}$ remain elastic. As the moment is increased further a plastic zone penetrates inwards to depth $h_{o}$, with or without hardening, as shown in Fig. 2b. Fibre AB is deformed elastically to its yield strain $\mathrm{A}^{\prime} \mathrm{B}^{\prime}$ and fibres $\mathrm{CD}$ and $\mathrm{EF}$ are deformed plastically to $\mathrm{C}^{\prime} \mathrm{D}^{\prime}$ and $\mathrm{E}^{\prime} \mathrm{F}^{\prime}$, Bending theory provides the total strain in fibre $\mathrm{EF}$, distance $\mathrm{y}$ from the neutral axis as:

$$
\varepsilon^{T}=\frac{E^{\prime} F^{\prime}-O^{\prime} O^{\prime}}{O^{\prime} O^{\prime}}=\frac{(R+y) \theta-R_{o} \theta_{0}}{R_{o} \theta_{0}}=\frac{\theta}{\theta_{o}}\left(\frac{R}{R_{0}}+\frac{y}{R_{o}}\right)-1
$$

As the neutral axis does not change its length then $\mathrm{O}^{\prime} \mathrm{O}^{\prime}=R_{o} \theta_{o}$ is the initial unstrained length of EF. Moreover, since $\mathrm{OO}=\mathrm{O}^{\prime} \mathrm{O}^{\prime}$

$$
R_{o} \theta_{o}=R \theta
$$

The radii of curvature $R_{o}$ and $R$ appear within the further strain expressions. In fibre CD the strain at yield is:

$$
\varepsilon_{Y}=\frac{Y_{o}}{E}=\frac{\left(R_{o}+d / 2\right) \theta_{o}-R_{o} \theta_{o}}{R_{o} \theta_{o}}=\frac{d}{2 R_{o}}
$$

Similarly, as the fibre $\mathrm{A}^{\prime} \mathrm{B}^{\prime}$ is strained to the yield point, we find $R$ from the inner elastic core:

$$
\varepsilon_{Y}=\frac{Y_{o}}{E}=\frac{\left(R+d / 2-h_{o}\right) \theta-R \theta}{R \theta}=\frac{\left(d / 2-h_{o}\right)}{R}
$$

Dividing eqs(3) and (4) gives the radius ratio: 


$$
\frac{R}{R_{0}}=1-\frac{2 h_{o}}{d}
$$

Substituting eqs(2) and (5) into eq (1) gives the total strain in fibre $\mathrm{E}^{\prime} \mathrm{F}^{\prime}$ as:

$$
\varepsilon^{T}=\frac{2 y \varepsilon_{Y}}{\left(d-2 h_{0}\right)}
$$

In the case of a non-hardening material (Fig. 2a) we find the plastic component of strain simply by subtracting $\mathcal{E}_{Y}=Y_{o} / E$ from eq(6). This gives:

$$
\begin{aligned}
\varepsilon^{T}-\varepsilon_{Y} & =\frac{2 y \varepsilon_{Y}}{\left(d-2 h_{0}\right)}-\varepsilon_{Y} \\
\frac{\varepsilon^{P}}{\varepsilon_{Y}} & =\frac{2 y}{\left(d-2 h_{0}\right)}-1
\end{aligned}
$$

In the case of a linear forward hardening material (Fig. 2b), the result in eq(7) allows the strain origin to be located at $\mathcal{E}_{Y}=Y_{o} / E$, so enabling the flow stress to be written in terms of total strain as:

$$
\sigma_{f}=Y_{o}+C_{f}^{T}\left(\mathcal{E}^{T}-\mathcal{E}_{Y}\right)
$$

Substituting from eq(6) into eq(8):

$$
\frac{\sigma_{f}}{Y_{o}}=1+\frac{C_{f}^{T}}{E}\left(\frac{2 y}{d-2 h_{o}}-1\right)=1+\frac{C_{f}^{P} / E}{1+C_{f}^{P} / E}\left(\frac{2 y}{d-2 h_{o}}-1\right)
$$

A similar flow stress is found using the plastic strain component as an abscissa:

$$
\frac{\sigma_{f}}{Y_{o}}=1+\frac{C_{f}^{P}}{Y_{o}} \varepsilon^{P}
$$

Comparing eq(9) with eq(10) we see that the forward plastic strain is correspondingly:

$$
\frac{\varepsilon^{P}}{\varepsilon_{Y}}=\frac{E}{E+C_{f}^{P}}\left[\frac{2 y}{\left(d-2 h_{0}\right)}-1\right]
$$

In the case of a Ludwick description of a non-linear forward hardening material:

$$
\frac{\sigma_{f}}{Y_{o}}=1+\left(\frac{\varepsilon^{P}}{\varepsilon_{o f}}\right)^{n_{f}}
$$


Equations (6) and (12) are combined to give:

$$
\frac{\varepsilon^{P}}{\varepsilon_{Y}}=\frac{2 y}{\left(d-2 h_{o}\right)}-\left[1+\left(\frac{\varepsilon^{P}}{\varepsilon_{o f}}\right)^{n_{f}}\right]
$$

from which $\mathcal{E}^{P}$ must be solved at the chosen position $y$.

\section{Model Materials}

Firstly, we shall compare a non-hardening with a bi-linearly hardening material in Figs 2a,b. The beam cross-section is rectangular of breadth $b$ and depth $d$ so that the neutral axis (n.a.) passes through the centre (see Fig. 1b). Let a pure elastic-plastic moment $M_{e p}$ be applied to produce tension above and compression below this axis with a depth of plastic penetration $h_{o}$ from the top and bottom edges Fig. $2 \mathrm{c}$.

\subsection{Elastic-Perfect Plasticity}

In the case of an elastic-perfectly plastic material (Fig.2a) the plastic penetration to depth $h_{o}$ occurs under a constant yield stress $Y_{o}$. The normalised residual stresses are given as [5]:

$$
\begin{gathered}
\frac{\sigma_{R}}{Y_{0}}=\frac{2 y}{d-2 h_{0}}-\frac{2 y}{d}\left[1+\frac{2 h_{0}}{d}\left(1-\frac{h_{0}}{d}\right)\right] \text { for } 0 \leq y \leq\left(\frac{d}{2}-h_{0}\right) \\
\frac{\sigma_{R}}{Y_{0}}=1-\frac{2 y}{d}\left[1+\frac{2 h_{0}}{d}\left(1-\frac{h_{0}}{d}\right)\right] \text { for }\left(\frac{d}{2}-h_{0}\right) \leq y \leq \frac{d}{2}
\end{gathered}
$$

where $y$ is the distance from the central neutral axis. The two terms in each equation correspond to subtracting the elastic stress recovered by moment release from the stress within the section under that moment. Figure $2 \mathrm{c}$ shows the two stress distributions graphically for $h_{o} / d=0.4$ and, from their difference, the residuals, projected as continuous lines in Fig. 2d. Equations (14a,b) describe the two linear branches in the residual stress plot for which the greatest value, $\sigma_{R} / Y_{o}=0.7$, lies at their intersection where $y=d / 2-h_{o}$. Note that the maximum elastic stress released $\sigma_{e}$ occurs at the outer edges in Fig. 2c, this being less that the available elastic range $2 Y_{o}$ in (a). Consequently, the loading-unloading process follows the path $\mathrm{OABC}$ in Fig. $2 \mathrm{a}$. 


\subsection{Linear Hardening}

Next consider, the case of bi-linear hardening (Fig. 2b) in which allowances are made for a Bauschinger effect, with a variable elastic range and different hardening rates under forward and reversed deformation. Loading-unloading follows the path OABCD where, to achieve a similar degree of stress release $\sigma_{e}$ shown, an elastic recovery from B to $\mathrm{C}$ is followed by a reversed flow from $\mathrm{C}$ to $\mathrm{D}$. Consequently, two plastic penetrations occur in the section: $h_{o}$ in forward flow and $h_{1}$ in reversed flow (Fig. 2e). To solve for $h_{1}$ the plastic tangent moduli are taken to be $C_{f}^{P}$ and $C_{r}^{P}$, these derive from $C_{f}^{T}$ and $C_{r}^{T}$ in Fig. $2 \mathrm{~b}$ as:

$$
C_{f, r}^{P}=\frac{C_{f, r}^{T}}{1-C_{f, r}^{T} / E} \quad \text { or } \quad C_{f, r}^{T}=\frac{C_{f, r}^{P}}{1+C_{f, r}^{P} / E}
$$

where $E$ is the elastic modulus.

\subsubsection{Elastic-Plastic Moments}

Firstly, an expression for $M_{e p}$ is required in forward loading along OAB. For this, the branched, stress distribution diagram above the neutral axis (continuous lines in Fig. 2e) is divided into three areas consisting of a rectangle and two triangles with key dimensions are $h_{o}, Y_{o}$ and $\sigma_{f}$ Given the beam width $b$, the force exerted at the centroid of each area can be found. These are mirrored in the areas below the neutral axis so that $M_{e p}$ becomes the sum of three couples:

$$
\begin{aligned}
1_{e p} & \left.=Y_{o} b h_{o} \times\left(d-h_{o}\right)+\frac{Y_{o} D}{2}\left(\frac{d}{2}-h_{o}\right) \times \frac{4}{3}\left(\frac{d}{2}-h_{o}\right)+\frac{\left(\sigma_{f}-Y_{o}\right) 0 n_{o}}{2} \times \mid d-\frac{L n_{o}}{3}\right) \\
& =Y_{o} b h_{o} d\left(1-\frac{h_{o}}{d}\right)+\frac{Y_{o} b d^{2}}{6}\left(1-\frac{2 h_{o}}{d}\right)^{2}+\frac{Y_{o} b d h_{o}}{2}\left(\frac{\sigma_{f}}{Y_{o}}-1\right)\left(1-\frac{2 h_{o}}{3 d}\right) \\
& =M_{Y_{0}}\left[1+2 D_{o}\left(1-D_{o}\right)+3 D_{o}\left(1-\frac{2 D_{o}}{3}\right)\left(\frac{\sigma_{f}}{Y}-1\right)\right]
\end{aligned}
$$

where $D_{o}=h_{o} / d$ and $M_{Y o}=Y_{o} b d^{2} / 6$ is the moment required to initiate yield $Y_{o}$ at the outer fibres. When $M_{e p}$ is released the path BCD is followed and the stress distribution diagram becomes the chain dotted line in Fig. 2e. Again, we take this to be composed of two limbs containing three areas with key dimensions $h_{1}, Y_{1}$ and $\sigma_{e}$ as shown. It follows $M_{e p}$ is also 
supplied by this diagram as:

$$
M_{e p}=M_{Y_{1}}\left[1+2 D_{1}\left(1-D_{1}\right)+3 D_{1}\left(1-\frac{2 D_{1}}{3}\right)\left(\frac{\sigma_{e}}{Y_{1}}-1\right)\right]
$$

in which $D_{1}=h_{1} / d$ and $M_{Y_{1}}=Y_{1} b d^{2} / 6$. Equating (16) and (17) gives:

$$
1+2 D_{0}\left(1-D_{0}\right)+3 D_{0}\left(1-\frac{2 D_{0}}{3}\right)\left(\frac{\sigma_{f}}{Y_{0}}-1\right)=\frac{Y_{1}}{Y_{0}}\left[1+2 D_{1}\left(1-D_{1}\right)+3 D_{1}\left(1-\frac{2 D_{1}}{3}\right)\left(\frac{\sigma_{\theta}}{Y_{1}}-1\right)\right]
$$

in which $Y_{1} \sigma_{f}$ and $\sigma_{e}$ are now to be found from the plastic surface strain.

\subsubsection{Elastic Ranges}

With the penetration to depth $h_{o}$ the path OAB is followed (Fig. 2b) and on release of $M_{e p}$ an immediate elastic recovery along $\mathrm{BC}$ will precede any reversed plastic penetration. The elastic range $\mathrm{BC}$, is bounded by the forward and reversed flow curves, these defining the reversed yield stress $Y_{1}$ as:

$$
\begin{aligned}
Y_{1}=\sigma_{f}-\sigma_{r} & =\left(Y_{o}+C_{f}^{P} \varepsilon^{P}\right)-\left(-Y_{o}+C_{r}^{P} \varepsilon^{P}\right) \\
& =2 Y_{0}+\left(C_{f}^{P}-C_{r}^{P}\right) \varepsilon^{P}
\end{aligned}
$$

in which $\mathcal{E}^{P}$ is the forward strain. Substituting eq(11) into eq(19):

$$
\frac{Y_{1}}{\bar{Y}_{0}}=2-\left(\frac{C_{r}^{P}-C_{f}^{P}}{E+C_{f}^{P}}\right)\left[\frac{2 y}{\left(d-2 h_{0}\right)}-1\right]
$$

in which $C_{r}{ }^{P}>C_{f}^{P}$. The question as to whether reversed plasticity occurs depends upon the magnitude of the elastic stress $\sigma_{e}$ that is driven by $M_{e p}$ 's release. Taking point B as the origin for the reversed flow curve allows the available elastic stress to be found from $M_{e p}$ :

$$
\frac{\sigma_{e}}{Y_{0}}=\frac{M_{e p}}{M_{Y_{0}}}=1+2 D_{0}\left(1-D_{0}\right)+3 D_{0}\left(1-\frac{2 D_{0}}{3}\right)\left(\frac{\sigma_{f}}{Y_{0}}-1\right)
$$

where $M_{Y_{o}}$ is the moment required to initiate yielding at the outer fibres. 


\subsubsection{Reversed Yielding}

A condition for reversed yielding (path CD) is therefore:

$$
\frac{\sigma_{e}}{Y_{0}}>\frac{Y_{1}}{Y_{0}}
$$

and then we may combine eqs(18) and (21) to find $D_{1}$ :

$$
\frac{\sigma_{\varepsilon}}{Y_{o}}=\frac{Y_{1}}{Y_{0}}\left\{1+2 D_{1}\left(1-D_{1}\right)+3 D_{1}\left(1-\frac{2 D_{1}}{3}\right)\left[\left(\frac{\sigma_{\varepsilon}}{Y_{0}}\right)\left(\frac{Y_{0}}{Y_{1}}\right)-1\right]\right\}
$$

Equation (23) may be solved for $D_{1}$ by setting:

leading to a quadratic:

$$
K=\frac{\sigma_{e} / Y_{o}}{Y_{1} / Y_{o}}
$$

$$
2 K D_{1}^{2}-(3 K-1) D_{1}+(K-1)=0
$$

to which the root is:

$$
D_{1}=\frac{K-1}{2 K}=\frac{1}{2}\left(1-\frac{Y_{1} / Y_{0}}{\sigma_{\varepsilon} / Y_{0}}\right)
$$

in which the two ratios $Y_{1} / Y_{o}$ and $\sigma_{e} / Y_{o}$ are given by eqs (20) and (21) respectively.

\subsubsection{Elastic Release}

On the other hand, the release will be purely elastic (between B and C in Fig. 2b) if

$$
\frac{\sigma_{e}}{Y_{0}} \leq \frac{Y_{1}}{Y_{0}}
$$

Within eq(25) the equality will supply a critical $h_{o}$ value when the release coincides with $\mathrm{C}$ :

$$
\frac{\sigma_{e}}{Y_{0}}=\frac{M_{e p}}{M_{Y_{0}}}=\frac{Y_{1}}{Y_{0}}
$$

where $M_{e p}$ and $Y_{1}$ are calculated from the outer fibre flow stresses $\sigma_{f}$ and $\sigma_{r}$. Substituting from eqs(20) and (21) with $D_{o}=h_{o} / d$ and $y=d / 2$ 


$$
1+2 D_{o}\left(1-D_{o}\right)+3 D_{\circ}\left(1-\frac{2 D_{o}}{3}\right)\left(\frac{\sigma_{f}}{Y_{o}}-1\right)=2\left[1-\left(\frac{C_{r}^{P}-C_{f}^{P}}{E+C_{f}^{P}}\right)\left(\frac{D_{o}}{1-2 D_{o}}\right)\right]
$$

Substituting $\sigma_{f} / Y_{o}$ from eq(9) into eq(26a) leads to a cubic equation in $D_{o}$ :

$$
1+2 D_{0}\left(1-D_{0}\right)+3 D_{0}\left(1-\frac{2 D_{o}}{3}\right)\left(\frac{2 D_{o}}{1-2 D_{0}}\right)\left(\frac{C_{f}^{P}}{E+C_{f}^{P}}\right)=2\left[1-\left(\frac{C_{r}^{P}-C_{f}^{P}}{E+C_{f}^{P}}\right)\left(\frac{D_{o}}{1-2 D_{o}}\right)\right]_{(26 b)}
$$

A solution to $D_{o}$ gives the critical value of initial penetration $h_{o}$, at or below which a reversed plasticity will not occur (i.e. $h_{1}=0$ ). When $h_{o}$ exceeds this critical value reversed plasticity occurs to a depth $h_{1}$, which may be found from eq(24c), as the following example shows.

\section{Example 1}

Let linear forward hardening (AB in Fig. 2b) be expressed in the gradient $C_{f}^{T} / E=1 / 10$ so that the plastic modulus becomes $C_{f}^{P}=1 / 9$, from eq(15a). Also, assume that the plastic moduli are in the ratio $C_{r}^{P} / C_{f}^{P}=3$. Substituting into eq(26b) leads to a cubic equation in $D_{o}$

$$
D_{o}^{3}-1.5 D_{o}^{2}+1.222 D_{0}-0.2777=0
$$

from which $D_{o}=0.333$. With initial penetrations beyond this critical value, the moment release will result in reversed yielding. Take, for example, $D_{o}=0.4$. The outer fibre $(y=d / 2)$ flow stress attained is, from eq(9):

$$
\frac{\sigma_{f}}{Y_{o}}=1+\frac{C_{f}^{P} / E}{1+C_{f}^{P} / E}\left(\frac{2 D_{o}}{1-2 D_{o}}\right)=1+\frac{1 / 9}{10 / 9}\left(\frac{0.8}{0.2}\right)=1.4
$$

and this corresponds to a normalised, forward plastic strain from eq(11) as:

$$
\frac{\varepsilon^{P}}{\varepsilon_{Y}}=\frac{1}{\left(1+C_{f}^{P} / E\right)}\left(\frac{2 D_{0}}{1-2 D_{0}}\right)=3.6
$$

The elastic range is, from eq(20):

$$
\frac{Y_{1}}{Y_{0}}=2-\left(\frac{C_{r}^{P} / E-C_{f}^{P} / E}{1+C_{f}^{P} / E}\right)\left(\frac{2 D_{o}}{1-2 D_{o}}\right)=2-\left(\frac{1 / 3-1 / 9}{1+1 / 9}\right)\left(\frac{0.8}{0.2}\right)=1.2
$$


The reversed flow stress is found from the elastic-plastic moment eq(21) to be:

$$
\frac{\sigma_{e}}{Y_{0}}=1+0.8(1-0.4)+1.2\left(1-\frac{0.8}{3}\right)(1.4-1)=1.832
$$

and this corresponds to a second penetration $D_{1}$ from eq(24c)

$$
D_{1}=\frac{1}{2}\left(1-\frac{1.2}{1.832}\right)=0.1724
$$

Each normalised quantity, when drawn to scale, reveals the two stress distributions superimposed in Fig. 2e: one corresponding to the moment application (continuous line) and the other to moment release (chain line). While the former shows two limbs, the latter appears to be linear (a consequence of linearity in CD). Taking the difference between their horizontal stress ordinates, i.e. the latter is subtracted from the former, provides the residual stress distribution given as the broken line in Fig. 2d. It is seen here that this does not differ appreciably from the residuals supplied by the non-hardening material model, corresponding to Fig. 1a and eqs $(14 a, b)$, where

$$
\frac{\sigma_{e}}{Y_{0}}=\frac{M_{Q p}}{M_{Y_{o}}}=1+2 D_{0}\left(1-D_{0}\right)=1.48
$$

\subsubsection{Special Case}

Many materials show little or no forward hardening. However, with a stress reversal the Bauschinger effect promotes a low yield stress and a sudden rapid rate of hardening. When such behaviour can be modelled by setting $C_{f}{ }^{P}=0$ and $C_{r}{ }^{P}=$ constant, the condition (26b) above becomes:

$$
1+2 \frac{h_{o}}{d}\left(1-\frac{h_{o}}{d}\right)=2\left[1-\frac{C_{r}^{P}}{E}\left(\frac{h_{o} / d}{1-2 h_{o} / d}\right)\right]
$$

leading to the cubic equation in the critical value of $h_{o} / d$ :

$$
4\left(\frac{h_{o}}{d}\right)^{3}-6\left(\frac{h_{o}}{d}\right)^{2}+\frac{2 h_{o}}{d}\left(2+\frac{C_{r}^{P}}{E}\right)-1=0
$$

The elastic range (eq 20) for unloading in the surface layers becomes 


$$
\frac{Y_{1}}{Y_{0}}=2-\left(\frac{C_{r}^{P}}{E}\right)\left(\frac{2 h_{0}}{d-2 h_{0}}\right)
$$

and since $\sigma_{f}=Y_{o}$ the elastic driving stress, eq(21), becomes

$$
\frac{\sigma_{\varepsilon}}{Y_{o}}=\frac{M_{e p}}{M_{\mathrm{Y}_{0}}}=1+\frac{2 h_{o}}{d}\left(1-\frac{h_{o}}{d}\right)
$$

which penetrates a second plastic zone to depth $h_{1}$ given by eq(24c) as:

$$
\frac{h_{1}}{d}=\frac{1}{2}-\left[\frac{1-\left(2+C_{r}^{P} / E\right) h_{o} / d}{\left(1+2 h_{o} / d\right)\left[1+2\left(1-h_{o} / d\right) h_{o} / d\right.}\right]
$$

\section{Example 2}

Taking $C_{r}^{T} / E=1 / 10$ gives $C_{r}^{P} / E=1 / 9$, this reducing eq(27) to give a critical initial penetration:

$$
4\left(\frac{h_{0}}{d}\right)^{3}-6\left(\frac{h_{o}}{d}\right)^{2}+4 . \dot{2}\left(\frac{h_{0}}{d}\right)-1=0
$$

the solution being $h_{o} / d=0.411$. Thus, if a deeper initial penetration is reached, say $h_{o} / d=$ 0.45 under a corresponding normalised plastic strain

$$
\frac{\varepsilon^{P}}{\varepsilon_{Y}}=\frac{2 D_{0}}{2 D_{0}-1}=9
$$

we find a second penetration to a depth $h_{1} / d=0.166$, given directly by eq(30). Alternatively, we find from eqs (28) and (29):

so that, from eq(24c):

$$
\begin{aligned}
& \frac{Y_{1}}{Y_{0}}=2\left[1-\frac{1}{9}\left(\frac{0.45}{1-0.9}\right)\right]=1 \\
& \frac{\sigma_{\varepsilon}}{Y_{0}}=1+0.9(1-0.45)=1.495
\end{aligned}
$$

$$
D_{1}=\frac{1}{2}\left(1-\frac{1}{1.495}\right)=0.166
$$


These "non-dimensions" are returned to their absolutes quantities within Fig.3a-d. The residuals appear as the shaded regions in Fig. 3c which are re-based in Fig. 3d. Again, a linear stress distribution applies to the release, this being a consistent feature of a linear hardening material in one or both directions. With $C_{f}{ }^{P}=C_{r}{ }^{P}=0$ for an elastic perfectlyplastic material, this stress release distribution is not altered. However, having $Y_{1} / Y_{o}=2$, there is no second penetration for the perfect material, but the residual stress distribution also remains unaltered.

\section{Hardening Material}

A similar reasoning is followed when extending the analyses given above to include parabolic hardening in both forward and reversed directions (Fig. 4a). The Ludwik law (12) is used to describe the "plastic" flow curve (Fig. 4b). Following Nadai [ ], the initial penetration appears with stress ordinates $Y_{o}$ and $\sigma_{f}$ in a mirror of the forward hardening (Fig. 4b). The elastic-plastic moment $M_{e p}$, is again found from three couples within the divided areas of this diagram. Noting that the area of a parabola is $2 / 3\left(\sigma_{f}-Y_{o}\right) h_{o}$ and the position of the centroid is $1 / 4 h_{o}$ from the top surface, $M_{e p}$ is found as

$$
\frac{M_{p p}}{M_{Y_{0}}}=1+2 D_{0}\left(1-D_{0}\right)+\frac{6}{n_{f}+1}\left[D_{0}\left(1-2 D_{0}\right)+\frac{2\left(n_{f}+1\right)}{\left(n_{f}+2\right)} D_{0}^{2}\right]\left(\frac{\sigma_{f}}{Y_{0}}-1\right)=\frac{\sigma_{\theta}}{Y_{0}}
$$

We have seen previously how, when written in this normalised form, equation (31) becomes the normalised elastic range (i.e. $\sigma_{e} / Y_{o}$ ) promoted by the moment release. The elastic range $Y_{1}$ (Fig. 4b) following a forward plastic strain is bounded by the forward and reversed flow curves as:

$$
\begin{aligned}
\frac{Y_{1}}{Y_{o}} & =\frac{\sigma_{f}}{Y_{o}}-\frac{\sigma_{r}}{Y_{o}}=\left[1+\left(\frac{\varepsilon^{P}}{\varepsilon_{o f}}\right)^{n_{f}}\right]-\left[-1+\left(\frac{\varepsilon^{P}}{\varepsilon_{o r}}\right)^{n_{r}}\right]=2+\left(\frac{\varepsilon^{P}}{\varepsilon_{o f}}\right)^{n_{f}}-\left(\frac{\varepsilon^{P}}{\varepsilon_{o r}}\right)^{n_{p}} \\
& =2+\left(\frac{\varepsilon_{Y}}{\varepsilon_{o f}}\right)^{n_{f}}\left(\frac{\varepsilon^{P}}{\varepsilon_{Y}}\right)^{n_{f}}-\left(\frac{\varepsilon_{Y}}{\varepsilon_{o r}}\right)^{n_{r}}\left(\frac{\varepsilon^{P}}{\varepsilon_{Y}}\right)^{n_{p}}
\end{aligned}
$$

where $f$ and $r$ refer the hardening constants $n$ and $\varepsilon_{o}$ to its respective direction. The coefficients $\mathcal{E}_{Y} / \mathcal{E}_{o f}$ and $\mathcal{E}_{Y} / \mathcal{E}_{o r}$ are assumed to be known (see example 3 ). The variable, the normalised plastic strain $\mathcal{E}^{P} / \mathcal{E}_{Y}$ in this equation is forward only, this being given from eq(13) as the solution to: 


$$
\left(\frac{\varepsilon_{Y}}{\varepsilon_{o f}}\right)^{n_{f}}\left(\frac{\varepsilon^{P}}{\varepsilon_{Y}}\right)^{n_{f}}+\frac{\varepsilon^{P}}{\varepsilon_{Y}}=\frac{2 D_{o}}{1-2 D_{0}}
$$

The critical $D_{o}$ value is found from equating (31) and (32). When $D_{o}$ exceeds this critical value the moment release results in a second plastic penetration to a normalised depth $D_{1}$. When $M_{e p}$ is derived from the corresponding stress distribution we have

$$
\frac{M_{a p}}{M_{Y_{1}}}==1+2 D_{1}\left(1-D_{1}\right)+\frac{6}{n_{r}+1}\left[D_{1}\left(1-2 D_{1}\right)+\frac{2\left(n_{r}+1\right)}{\left(n_{r}+2\right)} D_{1}^{2}\right]\left(\frac{\sigma_{\theta}}{Y_{o}}-1\right)
$$

Equating $M_{e p}$ between eqs(31) and (34) and noting that $M_{e p} / M_{\mathrm{Y}_{0}}=\sigma_{e} / Y_{o}$ and $M_{Y_{1}} / M_{Y_{0}}=Y_{1} / Y_{0}$ gives:

$$
\frac{\sigma_{e}}{Y_{0}}=\frac{Y_{1}}{Y_{0}}\left\{1+2 D_{1}\left(1-D_{1}\right)+\frac{6}{n_{r}+1}\left[D_{1}\left(1-2 D_{1}\right)+\frac{2\left(n_{r}+1\right)}{\left(n_{r}+2\right)} D_{1}^{2}\right]\left(\frac{\sigma_{e}}{Y_{0}}-1\right)\right\}
$$

Employing $K$ from eq(24a), eq(35) leads to the following quadratic equation in $D_{1}$ :

$$
\left[1+\frac{6(K-1)}{\left(2+n_{r}\right)\left(1+n_{r}\right)}\right] D_{1}^{2}-\left[1+\frac{3(K-1)}{\left(1+n_{r}\right)}\right] D_{1}-\frac{1}{2}(1-K)=0
$$

Figure $4 \mathrm{c}$ shows the two stress distributions with their absolute dimensions bounding the shaded residuals. When the latter are re-based in Fig. $4 \mathrm{~d}$ a non-linearity appears within the outer branches. This feature is responsible for the greatest deviation from the ideal material solution as the following example shows.

\section{Example 3}

Consider a rectangular beam in steel with $Y_{o}=250 \mathrm{MPa}, E=212.5 \mathrm{GPa}, n_{f}=0.2$ and $n_{r}=2$ that hardens to the extent that $\sigma_{f} / Y_{o}=1.5$ for a forward plastic strain of $1 \%$. Using a yield strain $\mathcal{E}_{Y}=Y_{o} / E=1.177 \times 10^{-3}$ this plastic strain is normalised to $\mathcal{E}^{P} / \mathcal{E}_{Y}=8.5$ for the flow curve $\mathrm{OAB}$ in Fig. 5a. A pronounced Bauschinger effect reveals $\sigma_{r} / Y_{o}=0.5$, as shown in point $\mathrm{C}$. This information allows our normalised Ludwik descriptions of the flow curves to be expressed as: 


$$
\begin{aligned}
& \frac{\sigma_{f}}{Y_{o}}=1+\left(\frac{\varepsilon_{Y}}{\varepsilon_{o f}}\right)^{n_{f}}\left(\frac{\varepsilon^{P}}{\varepsilon_{Y}}\right)^{n_{f}}=1+0.3259\left(\frac{\varepsilon^{P}}{\varepsilon_{Y}}\right)^{0.2} \\
& \frac{\sigma_{r}}{Y_{o}}=-1+\left(\frac{\varepsilon_{Y}}{\varepsilon_{o r}}\right)^{n_{r}}\left(\frac{\varepsilon^{P}}{\varepsilon_{Y}}\right)^{n_{F}}=-1+0.0208\left(\frac{\varepsilon^{P}}{\varepsilon_{Y}}\right)^{2}
\end{aligned}
$$

When a $1 \%$

plastic strain is reached at B on the beam surface, the depth of penetration $D_{o}=h_{o} / d$ is found from eq(33):

$$
0.3259\left(\frac{e^{P}}{\varepsilon_{Y}}\right)^{0.2}+\frac{\varepsilon^{P}}{\varepsilon_{Y}}=\frac{2 D_{0}}{1-2 D_{0}}
$$

which gives $D_{o}=0.45$. A a second penetration will occur from releasing the applied moment if $D_{o}=0.45$ exceeds a critical value. The critical $D_{o}$ is found equating (31) and (32) whilst ensuring that eq(33) holds. Thus, we assume a value for $D_{o}$ and substitute this into eq(33) to find $\mathcal{E}^{P} / \mathcal{E}_{Y}$ and then check for the required equality between eqs (31) and (32). The value $D_{o}$ $\simeq 0.41$ is found to satisfy both conditions. It is located at point A on the flow curve in Fig. 5a, where:

$$
\frac{\varepsilon^{P}}{\varepsilon_{Y}}=4.31, \frac{\sigma_{f}}{Y_{0}}=1.436\left(=\frac{\sigma_{A}}{Y_{0}}\right), \frac{Y_{1}}{Y_{0}}=\frac{\sigma_{e}}{Y_{0}}=2.05\left(=\frac{Y_{A}}{Y_{0}}=\frac{\sigma_{A}^{e}}{Y_{0}}\right)
$$

These dimensions re-appear in Fig. 5b within the two, tensile-side, stress distributions: (i) under the applied moment (continuous line) and (ii) the critical elastic release (chain line). The residual stress (Fig. 5c) is then derived from the difference between their stress levels. Once again, despite the allowance made for hardening the residuals differ only marginally from the non hardening solution (broken line).

Returning to point $\mathrm{B}$, where $D_{o}=0.45$ exceeds our critical value, the depth of a second penetration $D_{1}$ is found from eq(35) where:

$$
n_{r}=2, \frac{\sigma_{f}}{Y_{o}}=1.5, \frac{Y_{1}}{Y_{o}}=1, \frac{\sigma_{s}}{Y_{o}}=2.16, K=\frac{\sigma_{\varepsilon} / Y_{o}}{Y_{1} / Y_{o}}=2.16
$$


from which the quadratic eq(36) becomes

$$
1.58 D_{1}^{2}-2.16 D_{1}+0.58=0
$$

for which the roots are $D_{1}=0.367$ and 1 . The second root of unity rests solely upon this choice of $n_{r}$ and should be disregarded, with it being greater than 0.5 . The two $D$ 's are shown in their stress distribution diagrams in Fig. $5 \mathrm{~d}$ : $D_{o}$ under the applied moment and $D_{1}$ following its release. Here, in contrast to the linear hardening model, the influence of non linear hardening appears within both stress distributions. For a given $D_{o}$, eq(13) is applied as follows to solve the plastic strain $\mathcal{E}^{P} / \mathcal{E}_{Y}$ in any intermediate fibre with position $y / d$.

$$
\frac{\varepsilon^{P}}{\varepsilon_{Y}}+\left(\frac{\varepsilon_{Y}}{\varepsilon_{o f}}\right)^{n_{f}}\left(\frac{\varepsilon^{P}}{\varepsilon_{Y}}\right)^{n_{f}}=\frac{2(y / d)}{\left(1-2 D_{o}\right)}-1
$$

from which eqs $(37 \mathrm{a}, \mathrm{b})$ supply the stress in that fibre. Each distribution in Fig. 5d replicates its respective hardening curve which, through their subtraction, influence the residual stress distribution as shown in Fig. 5e. Here, in the comparison made with the ideal material (broken line) the influence of reverse yielding upon residual stress is seen to have its greatest effect.

\section{Discussion}

The previous example reveals that residual stresses are influenced by non-linear hardening, with an allowance made for a Bauschinger effect that permits a second penetration. There are however, still further refinements that could be made to this model. For example, a reversed flow curve will depend upon the degree of forward plastic strain. There are any number of reversed flow curves $C D$ corresponding to the single forward flow curve $A B$ though the former do converge into a single curve as the reversed strain accumulates. In an extension to the present report we shall account for this behaviour by locating a new origin for reversed flow at point $B$ so allowing curve $C D$ to be derived from curve $A B$ by a suitable scheme. This becomes an important consideration, when in practice one would not have available the reversed flow curve corresponding to the forward strain imparted by a plastic penetration. 


\section{Conclusions}

The ideal elastic-perfectly plastic material model provides only a first approximation to elastic-plastic bending of beams. The zone of plasticity penetrates under a constant yield stress, recovery from moment release is purely elastic and the residuals are composed of linear branches. A linear hardening model allows the zone to penetrate with an increasing flow stress. Moment release will reveal a second penetration though the residual stress distribution is little altered from that of the ideal material. A more realistic theoretical account lies within the application of equilibrium and compatibility principles to a non-linear hardening material model. The stress distributions reflect forward hardening and a Bauschinger effect where it accompanies the second penetration with reversed plasticity from the moment release. Consequently, the residuals that account for non-linear hardening behaviour differ from simpler material models and are believed to be nearer to the true distribution.

\section{References}

[1] Bauschinger, J. Ueber die Veranderung der Elasticitatagrenze und dea Elasticitatamoduls verschiadener Metalle, Zivilingenieur, 1881, 27, 289-348.

[2] Svensson,N.L. Anisotropy and the Bauschinger effect in cold rolled aluminium, $J l$ Mech Eng Sci, 1966, 8, 162-172.

[3] Milligan,R.V, Koo,W.H. and Davidson,T.E. The Bauschinger effect in a high strength steel, Trans ASME, D, 1966, 480-488.

[4] Rees,D.W.A. Anisotropic hardening theory and the Bauschinger effect, Jl Strain Analysis, 16(2) 1981, 85-95.

[5] Rees, D.W.A. Mechanics of Solids and Structures, 2000, I.C. Press, World Scientific.

[6] Nadai, A. Theory of Flow and Fracture of Solids, 1950, McGraw-Hill, London. 


\section{List of Figures}

Fig. 1 Beam curvatures (a) when the yield stress $Y_{o}$ is reached at the outer fibres and (b) for a plastic penetration to depth $h_{o}$ from top and bottom surfaces.

Fig. 2 Moment application and release. Diagrams (a) and (c) refer to an elastic-perfectly plastic material, (b) and (e) to a linearly hardening material with reversed yielding to D. Geometrical interpretations of each material's residual stress distributions is shown in (d) where (- - - -) is for material (a) and (__ $)$ for material (b).

Fig. 3 Mixed mode material in which no forward hardening occurs for an abscissa of (a) total strain and (b) plastic strain. Shaded region in (c) defines horizontal ordinates of residual stress that have been re-based in (d). Despite reversed yielding solution in (d) is identical to that found from elastic-perfectly plastic material.

Fig. 4 (a) Non-linear hardening, defined by Ludwik's law for an abscissa of plastic strain in (b). Shaded region in (c) defines residual stress (re-based in d) when reversed yielding occurs with moment release to depth $h_{1}$.

Fig. 5 (a) A Ludwik non-linear hardening beam material showing two levels of plasticity for initial penetrations $h_{o}$. Point A corresponds to a critical penetration in which moment release is elastic. Residuals in (c) are derived from (b) as shown. Moment release from point B is elastic-plastic resulting in a second penetration $h_{1}$. Residuals in (e), as derived from (d), show their greatest departure from those of an ideal non-hardening material (- - - -). 
Figure(s)

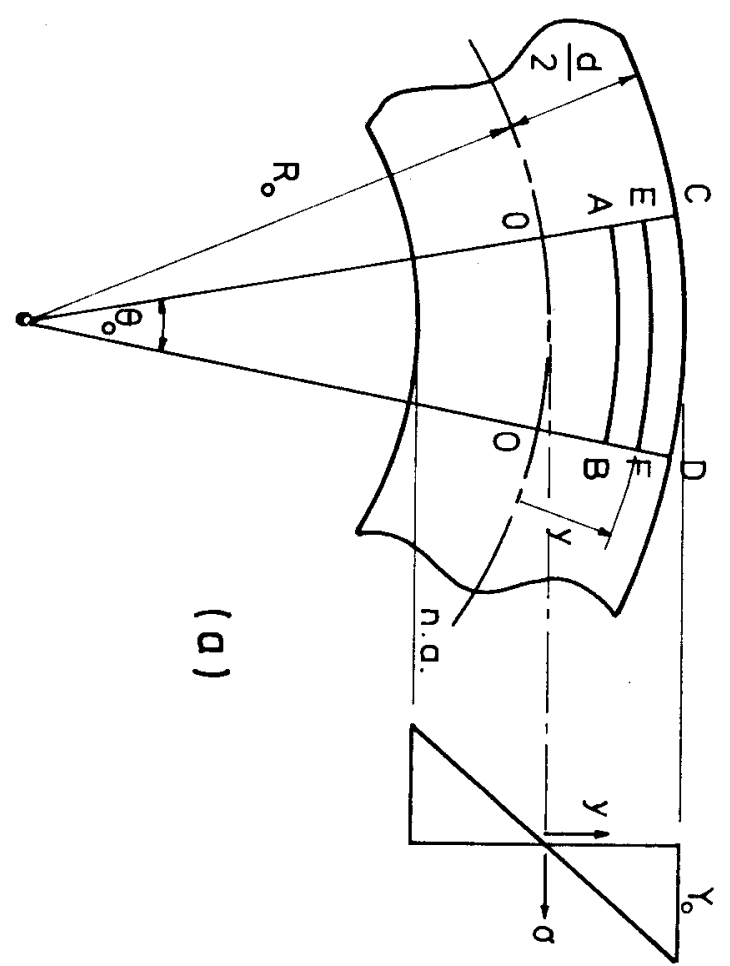

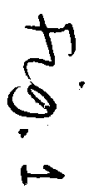

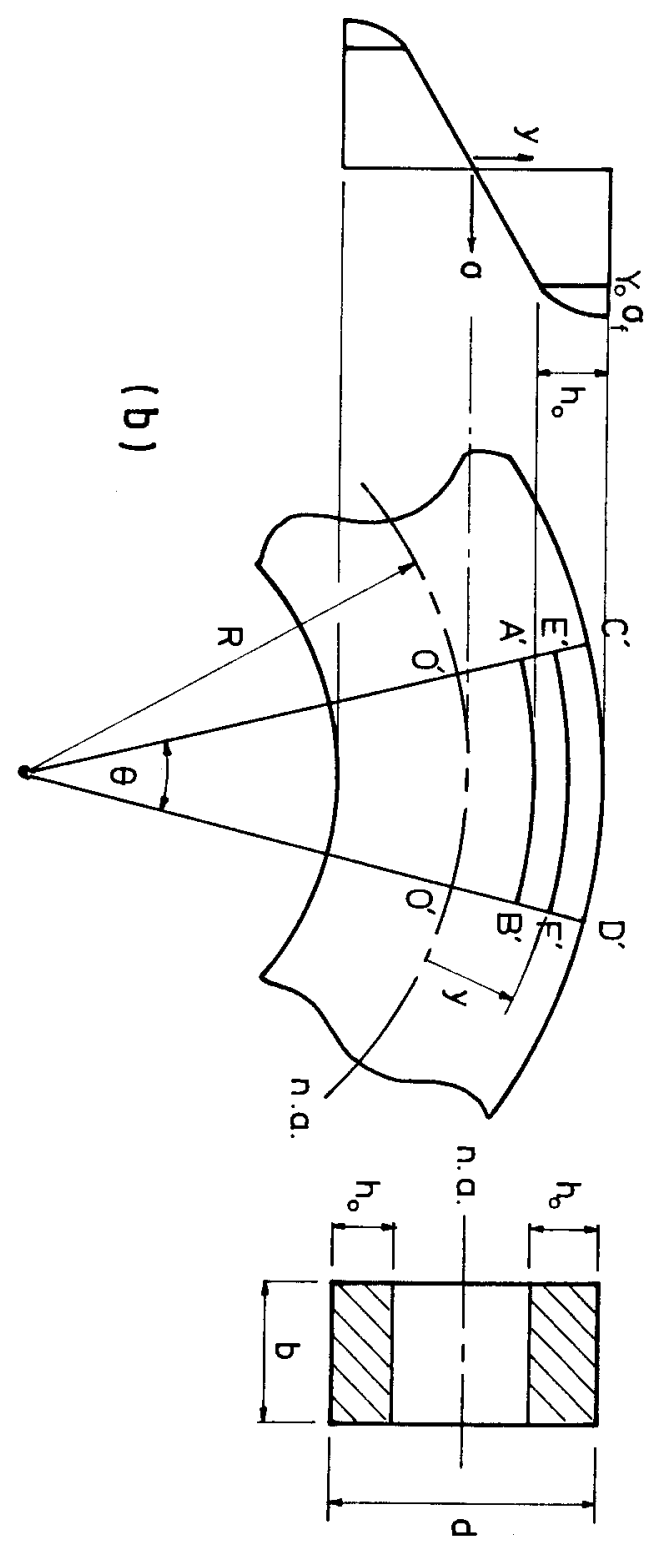



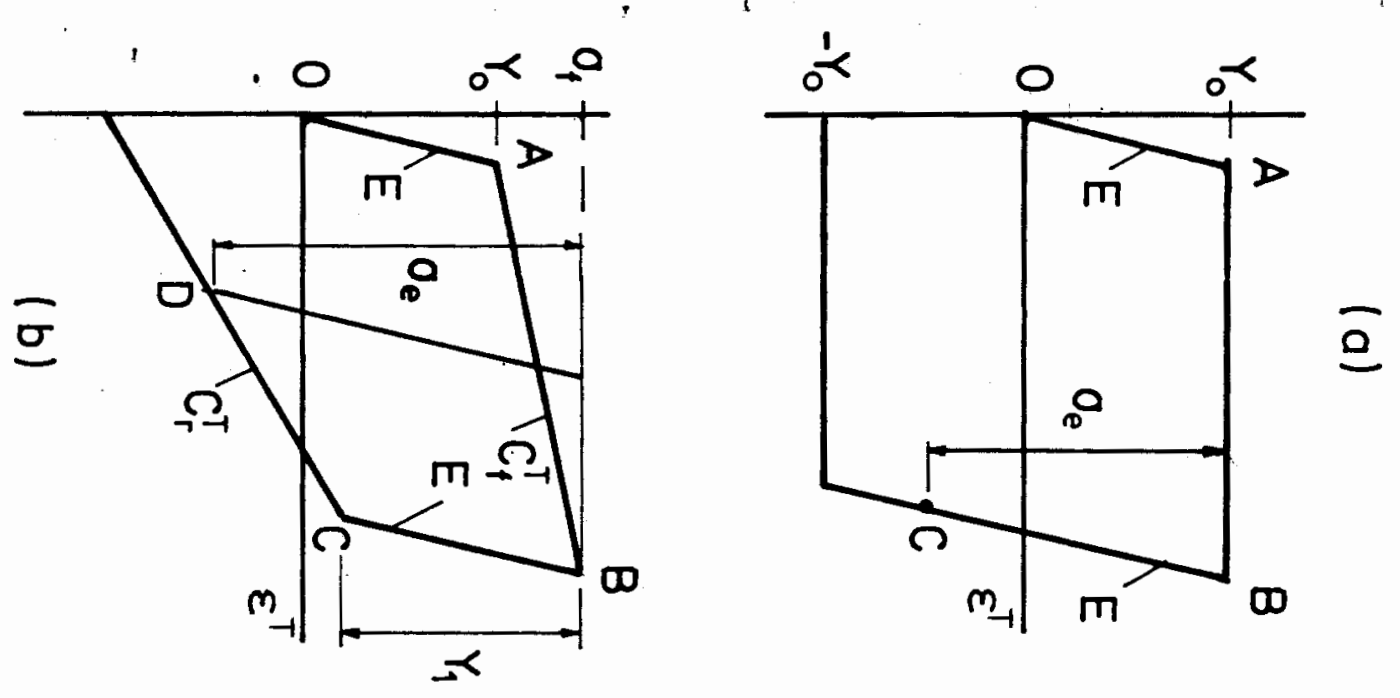

ग)

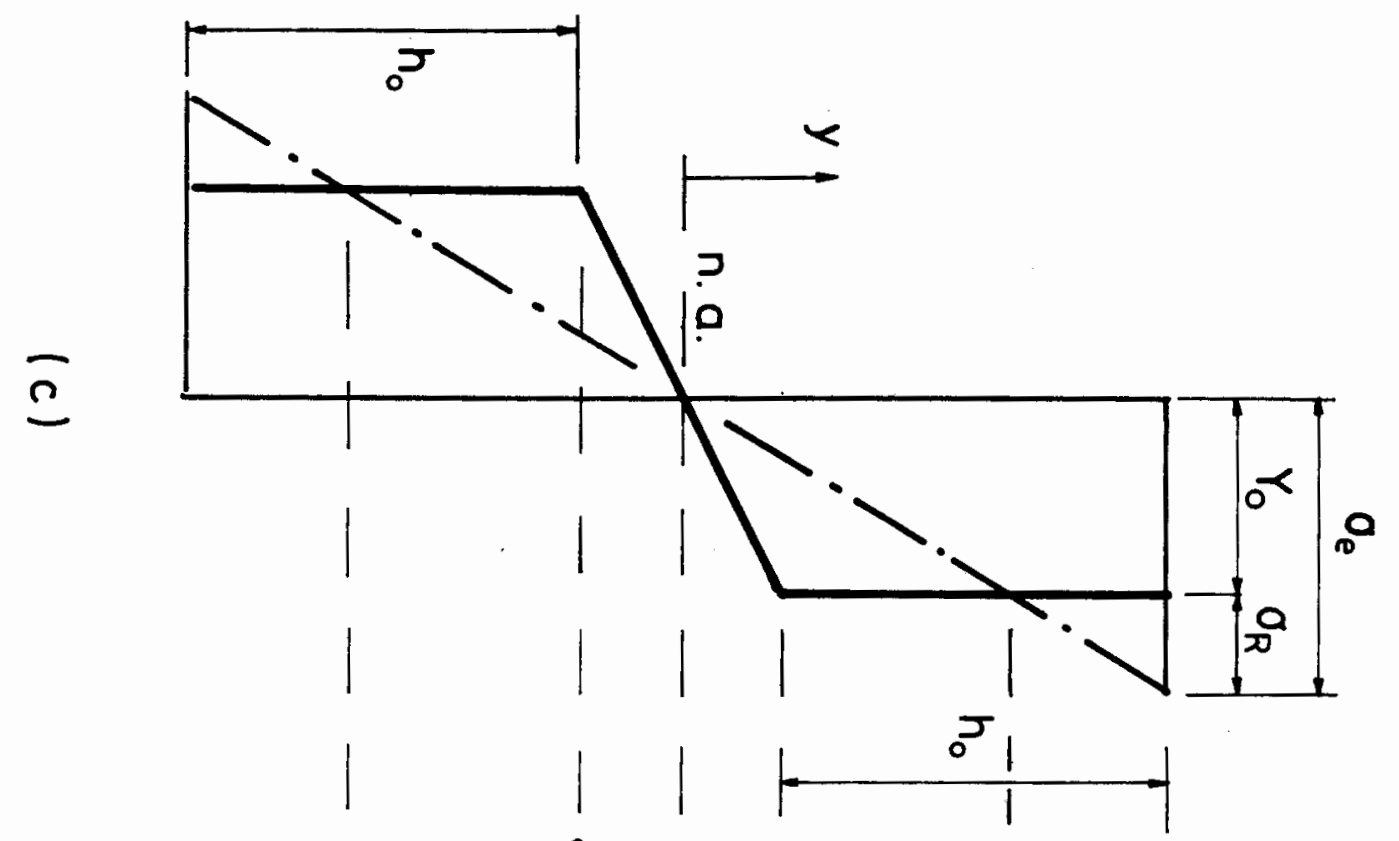

믐

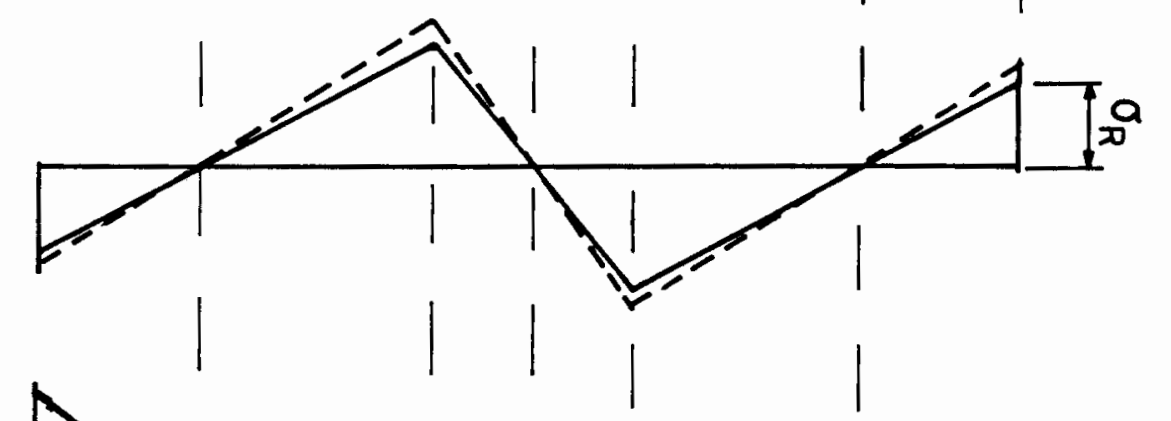

- 


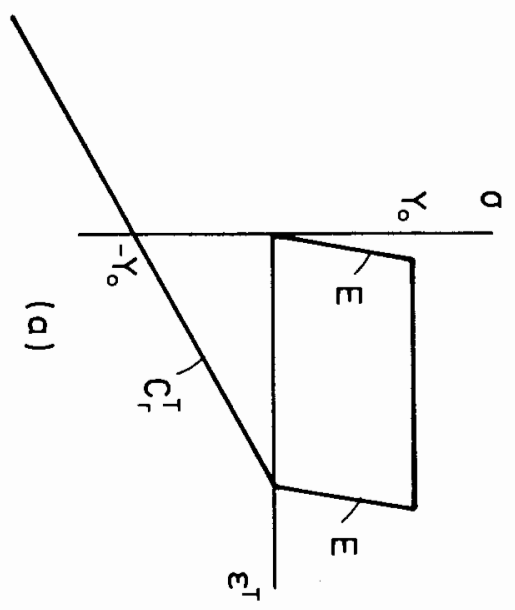

3

w
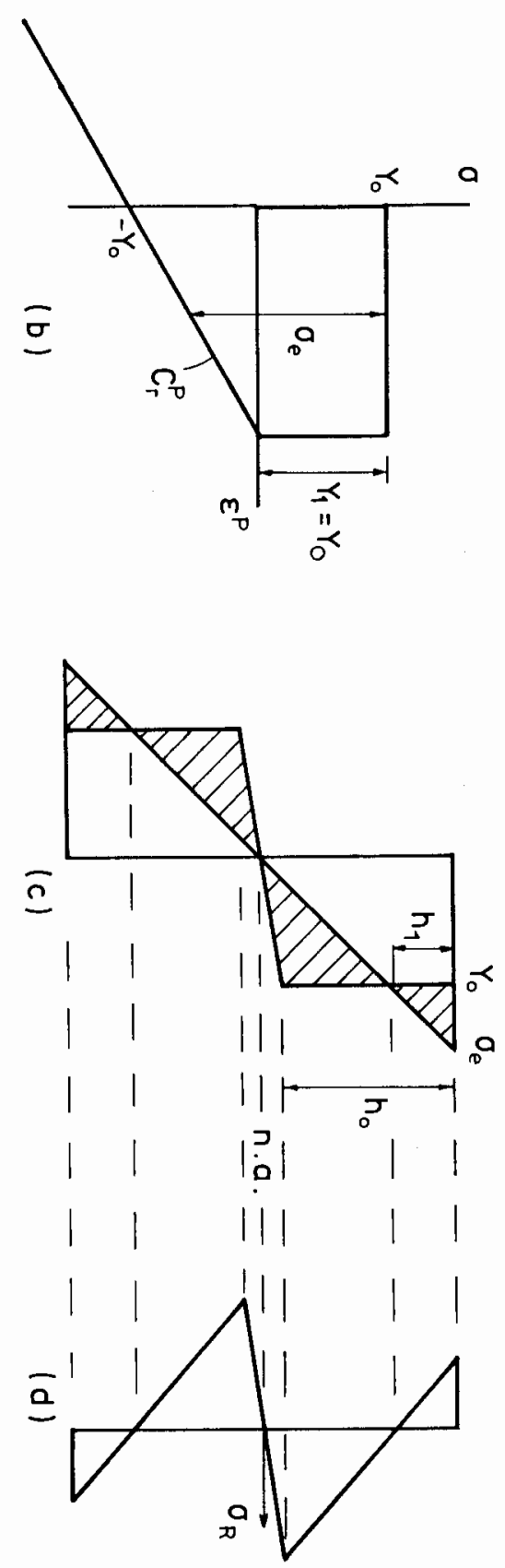


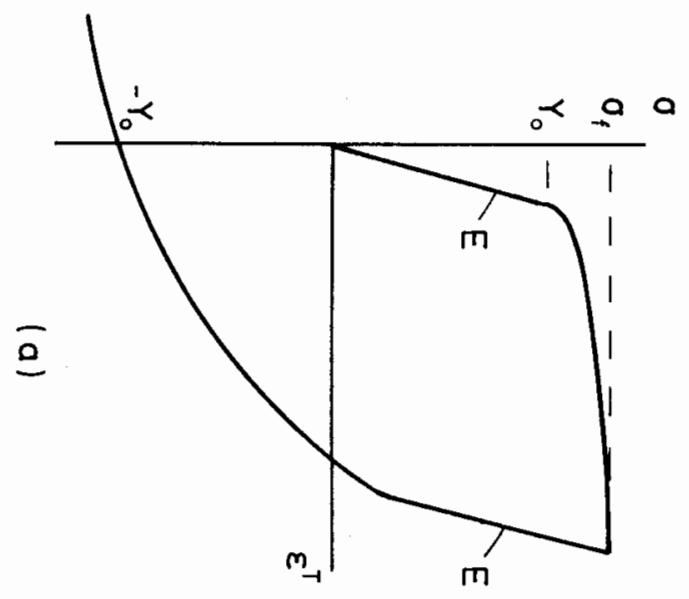

जी

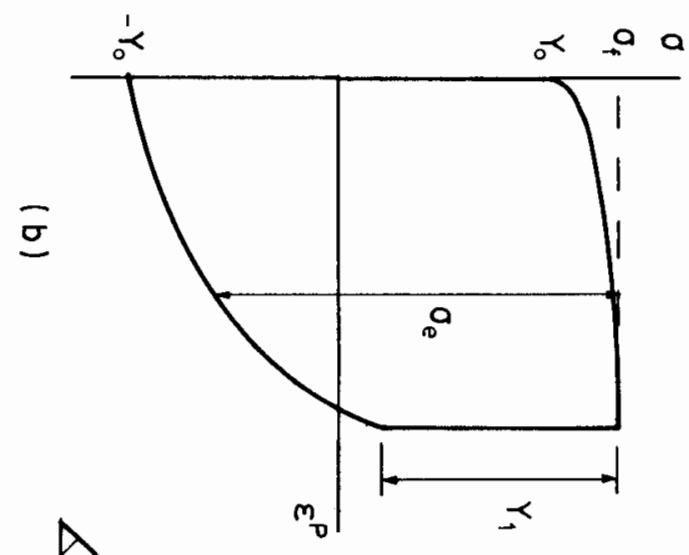

$\triangle$

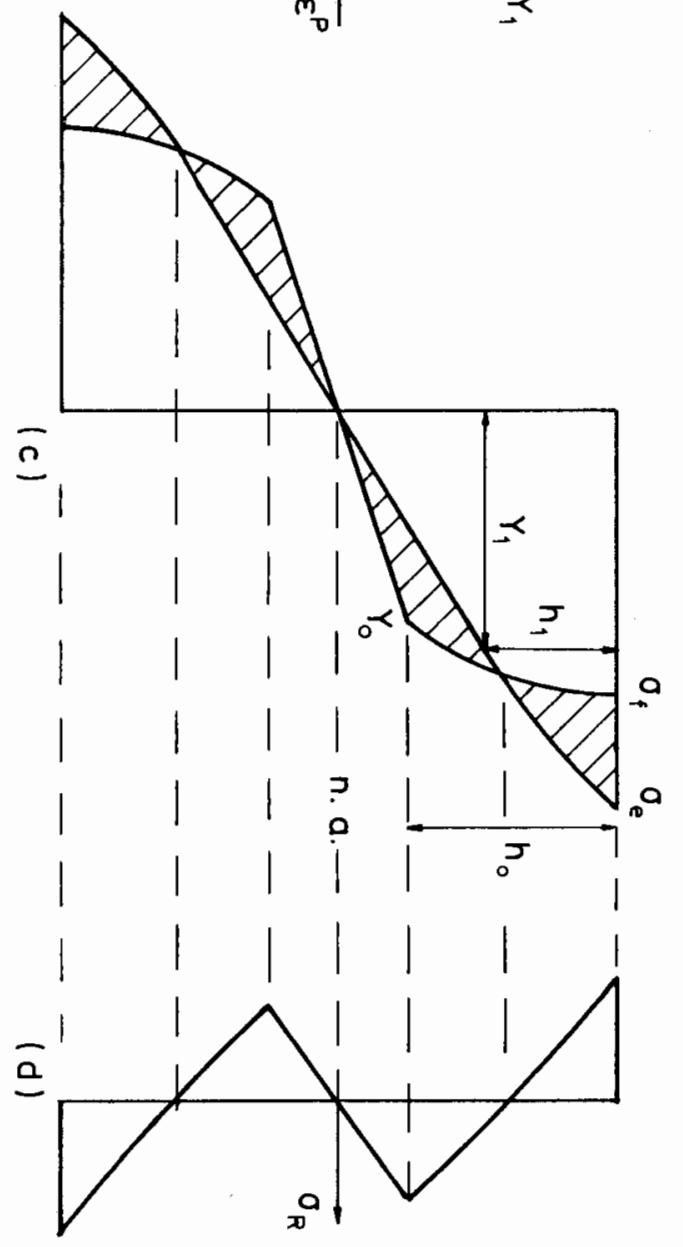




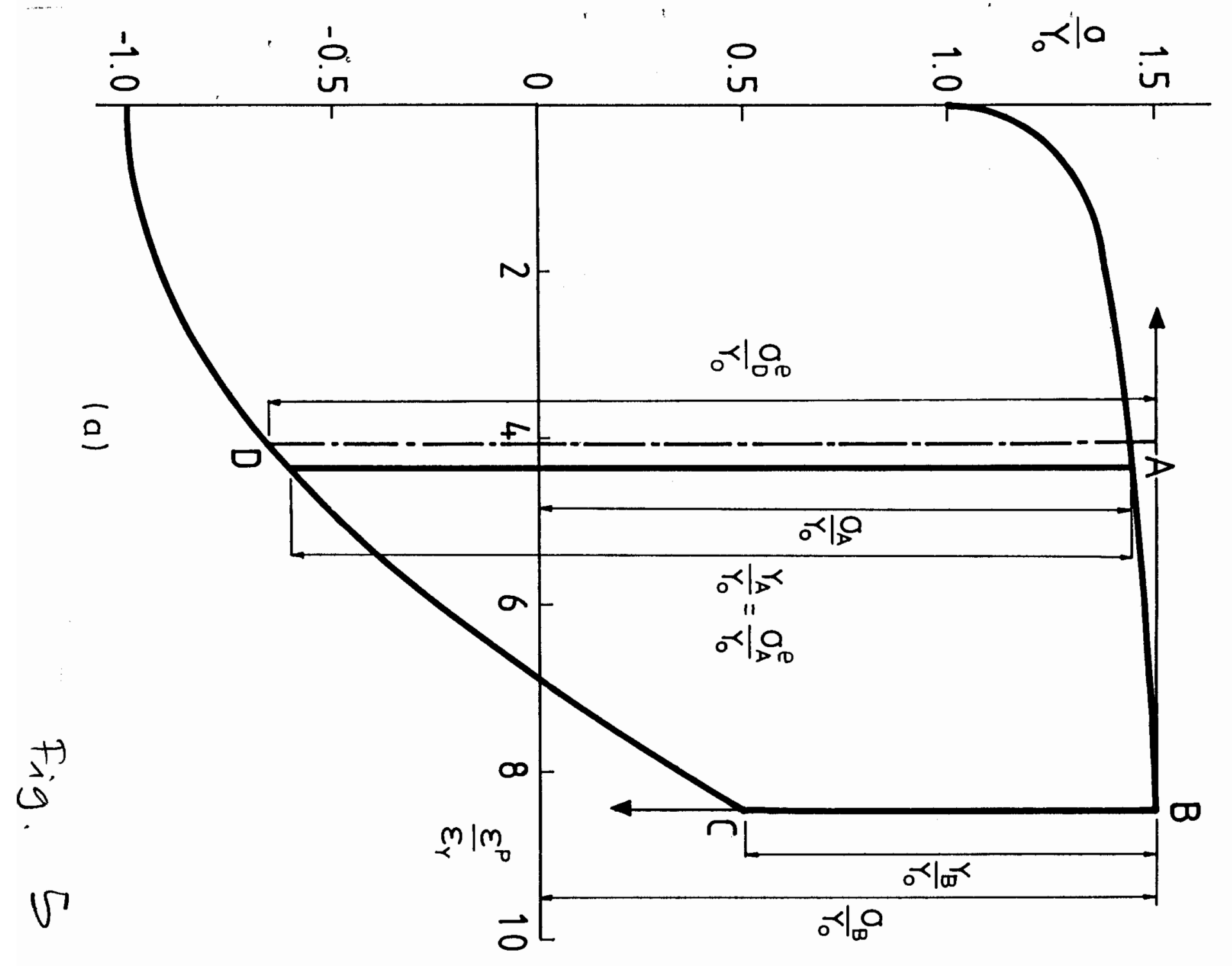

$\vec{t}^{+}$
$\vec{v}$
$v$
미
이

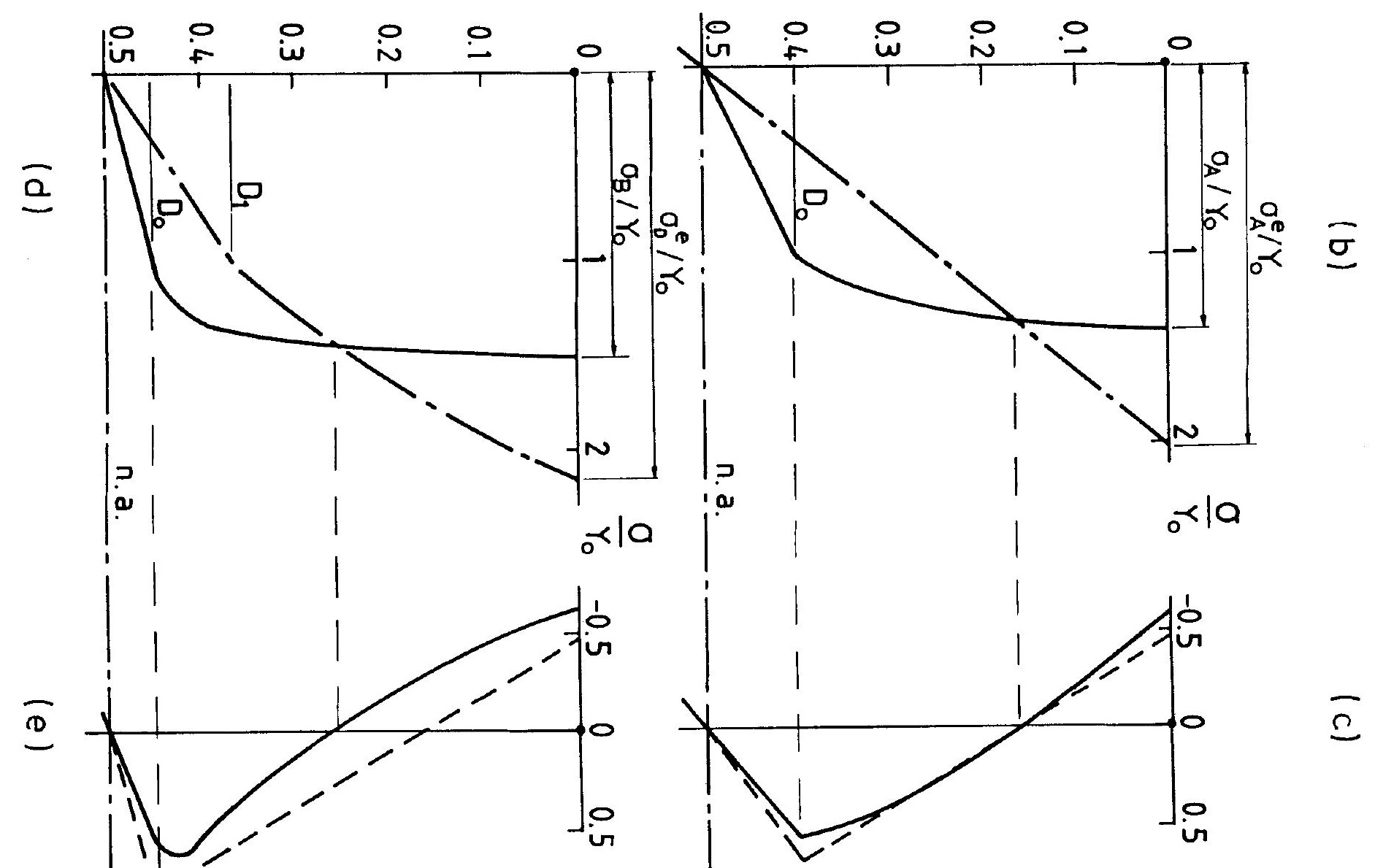

